\title{
An adolescent with polycystic ovary syndrome
}

\author{
Marja Ojaniemi and Michel Pugeat ${ }^{1}$ \\ Department of Pediatrics, Biocenter Oulu, University of Oulu, Oulu, Finland and ${ }^{1}$ Department of Endocrinology, East Pole of Lyon Hospitals, and INSERM \\ Unit 0322, Lyon, France \\ (Correspondence should be addressed to M Ojaniemi; Email: marja.ojaniemi@oulu.fi)
}

\begin{abstract}
Polycystic ovary syndrome (PCOS) is a common clinical condition that manifests during adolescence with menstrual irregularities, acne, and hirsutism. As these symptoms are frequently observed in healthy teenagers, it can be difficult to recognize PCOS. Establishment of hyperandrogenism, polycystic ovaries, and identifying a metabolic disorder are required for the management of PCOS in a teenager. The underlying defects in PCOS are still unclear; however, insulin resistance and the metabolic syndrome are common in both obese and non-obese PCOS patients, so that the evaluation of glucose tolerance is recommended. More than $50 \%$ of PCOS patients are overweight or obese, and will benefit from an increase in physical activity and weight loss. Metformin is a treatment option that requires further investigation before being recommended on a long-term basis.
\end{abstract}

European Journal of Endocrinology 155 S149-S152

\section{Introduction}

Polycystic ovary syndrome (PCOS) in a teenager is characterized by irregular menstrual cycles, generally less than six menses per year, and by clinical or biochemical features of hyperandrogenism. More than $50 \%$ of PCOS patients have the metabolic syndrome, including obesity, insulin resistance, and dyslipidemia (1). Although PCOS is a common disorder, the diagnosis may be overlooked during adolescence, as irregular menses with anovulatory cycles, obesity, and acne are frequent in adolescent women. According to the androgen overexposure hypothesis, PCOS may have its origin already in fetal life, but becomes clinically manifest during adolescence with maturation of the hypothalamic-pituitaryovarian axis (2). The incidence of PCOS among adolescents is estimated to be between 11 and 26\% (3) and about $50 \%$ are overweight. The pathophysiology of PCOS is still uncertain, although there is evidence that both genetic and environmental factors may play a role, resulting in ovarian hyperandrogenism and impaired insulin sensitivity (4-8). As the underlying defects for PCOS are unclear, the management of PCOS in teenage girls is symptomatic. Weight control and physical activity reduce hyperandrogenism and improve insulin sensitivity (9). Metformin has been shown in several studies to improve insulin sensitivity, lower androgen levels, and to induce menstrual cyclicity in adults $(10,11)$. However,

This paper was presented at the 4th Ferring Pharmaceuticals International Paediatric Endocrinology Symposium, Paris (2006). Ferring Pharmaceuticals has supported the publication of these proceedings. there are limited data regarding the use of metformin in the treatment of adolescents with PCOS and impaired glucose tolerance $(6,9,12-15)$.

Here, we report a case of an adolescent girl with PCOS, who has been treated with metformin for 4.5 years.

\section{Case report}

\section{Patient}

In September 2001, a 15.5-year-old girl presented for evaluation of obesity and amenorrhea.

The patient was the third child in the family. Her parents were of normal weight, and they both had hypertension, hypercholesterolemia, and impaired glucose tolerance. Her mother had gestational diabetes mellitus during her pregnancies. Two female siblings were healthy and of normal weight.

The patient started a marked weight gain from 8 years of age. Over the next 7 years, her weight increased from the 10th to 70th centile by the time of presentation. Her height SDS remained consistent at -0.5 S.D. Menarche occurred at the age of 14, but she soon thereafter she developed secondary amenorrhea.

\section{Assessment}

The patient was obese; her height was $160.8 \mathrm{~cm}(-0.5$ S.D.); weight $83.0 \mathrm{~kg}$ (71 centile), body mass index (BMI) $32.1 \mathrm{~kg} / \mathrm{m}^{2}$, waist circumference $85 \mathrm{~cm}$, and blood pressure 135/84. Her pubertal stage was Tanner B4, 
P4. The patient was hirsute (Ferriman-Gallway score 12) and had mild acne. She had no acanthosis nigricans.

Serum testosterone and luteinizing hormone levels were elevated, while follicle-stimulating hormone, estradiol, and 17-OH progesterone levels remained normal (Table 1). Baseline serum thyroid-stimulating hormone, free thyroxine, prolactin, and 24-h free urinary cortisol were within normal limits. Serum transaminases and creatine were normal.

The patient had a slightly elevated fasting blood glucose with an abnormal increase during an oral glucose tolerance test (OGTT), indicating impaired glucose tolerance. She also had severe hyperinsulinemia throughout the test (Table 2). The serum lipid profile was abnormal with elevated total cholesterol, low density lipoprotein (LDL)-cholesterol, triglycerides levels, and low high density lipoprotein (HDL)-cholesterol levels respectively (Table 2).

Transrectal pelvic ultrasound showed bilateral polycystic ovaries (Fig. 1).

Secondary amenorrhea with hirsutism and an elevated testosterone level, together with the appearance of polycystic ovaries on ultrasound established the diagnosis of PCOS. She was evaluated by a nutritional therapist and a physiotherapist and given lifestyle advice to reduce weight and increase physical activity. To improve her glucose tolerance and induce menstruation, metformin $1.5 \mathrm{~g} /$ day $(750 \mathrm{mg} \times 2$ p.o.) and dydrogesterone (days 15-24 of the menstrual cycle) were started in order to improve glucose tolerance and induce menses.

Within 6 months, there was a $4.5 \mathrm{~kg}$ weight reduction and her BMI decreased to $30.3 \mathrm{~kg} / \mathrm{m}^{2}$. Glucose tolerance improved, hyperinsulinemia ameliorated and there was some improvement in the lipid profile (Table 2). The LDL-receptor was screened for mutations in view of a family history of hypercholesterolemia; the results were negative. Menses occurred only after dydrogesteroneinduced withdrawal; metformin was increased to $2.25 \mathrm{~g} /$ day $(750 \mathrm{mg} \times 3$ p.o.). After 1 year on treatment, weight was $70.8 \mathrm{~kg}$ ( 45 centile) and BMI, $27.3 \mathrm{~kg} / \mathrm{m}^{2}$. Fasting glucose and insulin levels were normal and the lipid profile was further improved. Polycystic ovaries remained but menses were now normal ovulatory menstrual cycles without progesterone medication.

Presently, 4.5 years after initial presentation, the patient remains on metformin therapy. She is still obese and has problems in controlling her weight. However, glucose and insulin levels have remained normal as assessed by yearly OGTTs. She also has normal

Table 1 Baseline serum gonadotropin, testosterone, estradiol, and 17-OH progesterone levels.

\begin{tabular}{lcc}
\hline & Serum & Reference range \\
\hline Follicle-stimulating hormone $(\mathrm{U} / \mathrm{l})$ & 5.9 & $3.3-6.06$ \\
Luteinizing hormone $(\mathrm{U} / \mathrm{l})$ & 11.0 & $4.8-10.73$ \\
Testosterone $(\mathrm{nmol} / \mathrm{l})$ & 4.3 & $0.42-2.05$ \\
Estradiol (nmol/l) & 0.18 & $0.143-0.324$ \\
17-OH progesterone $(\mathrm{nmol} / \mathrm{l})$ & 2.8 & $<4.4$ \\
\hline
\end{tabular}

ovulatory cycles and showed normal morphology of the ovaries on a recent ultrasound scan.

\section{Discussion}

The Rotterdam 2004 Consensus Workshop (16) proposed that PCOS is a syndrome of ovarian dysfunction, and recommended that two of the following criteria should be present to establish a diagnosis: chronic oligo- or anovulation for more than 6 months, clinical and/or biochemical evidence of hyperandrogenism, and polycystic ovaries on ultrasound. Other disorders that mimic the PCOS phenotype should be excluded. The criteria for PCOS were fulfilled in this patient. She was amenorrhoic and had clinical and biochemical signs of hyperandrogenemia and polycystic ovaries on ultrasound. Gonadotropin and estradiol levels were normal, thus excluding primary ovarian failure or hypo- or hypergonadotropic hypogonadism. Hyperprolactinemia and late-onset congenital adrenal hyperplasia were excluded because of normal prolactin and $17-\mathrm{OH}$ progesterone levels. Furthermore, there was no evidence of hypercortisolism and thyroid disease. PCOS is generally underdiagnosed during adolescence. While clinical and metabolic features are similar to those in adults, it can be difficult to distinguish the young PCOS woman from features seen in a normal adolescent. PCOS has been detected in 95\% of adolescents with irregular menses (17). Many are treated with the oral contraceptive pill, which may mask the condition until pregnancy is attempted (2). Clinicians should note that abnormal menses, such as cycles shorter than 21 days or longer than 35 days, are often associated with PCOS.

The spectrum of PCOS phenotype is wide; this includes women with no evidence of clinical and biochemical hyperandrogenism despite dysfunctional polycystic ovaries. Our patient had mild symptoms of hyperandrogenemia and the serum testosterone level was elevated (18). Testosterone is the principal circulating androgen in women, produced from ovarian and adrenal precursors (mainly androstenedione and dehydroepiandrostenedione), metabolized in peripheral tissues. Some adolescent PCOS patients may have normal androgen levels (19) with moderate hirsutism compared to adults with PCOS (20). Measurement of total testosterone using a reliable immunoassay is recommended to establish hyperandrogenemia. Androgen-secreting tumors are rare in adolescents but need to be excluded. A marked increase in serum testosterone $(8.7 \mathrm{nmol} / \mathrm{l}$, two to three times the upper normal range), with a normal DHEA-sulfate (DHEAS) level, is highly suggestive of an androgen-secreting ovarian tumor. When DHEAS is also increased $(>16.3 \mu \mathrm{mol} / \mathrm{l})$, this suggests an adrenal androgen-secreting tumor.

Insulin resistance plays an important role in PCOS physiopathology. During puberty, insulin sensitivity is 
Table 2 Oral glucose tolerance test (OGTT) and lipid profiles before and after therapy.

\begin{tabular}{|c|c|c|c|c|c|c|}
\hline \multirow[b]{2}{*}{$\begin{array}{l}\text { OGTT (h) } \\
\text { Glucose (mmol/l) } \\
\text { Insulin (mU/l) }\end{array}$} & \multicolumn{3}{|c|}{ Pre-treatment } & \multicolumn{3}{|c|}{ Post-treatment (6 months) } \\
\hline & $\begin{array}{l}0 \\
6.1 \\
37.0\end{array}$ & $\begin{array}{c}1 \\
10.2 \\
113.6\end{array}$ & $\begin{array}{r}2 \\
9.1 \\
117.8\end{array}$ & $\begin{array}{c}0 \\
5.9 \\
23.4\end{array}$ & $\begin{array}{c}1 \\
8.2 \\
62.3\end{array}$ & $\begin{array}{c}2 \\
8.0 \\
74.7\end{array}$ \\
\hline Lipid profile & & \multicolumn{2}{|c|}{ Pre-treatment } & \multicolumn{3}{|c|}{ Post-treatment (6 months) } \\
\hline $\begin{array}{l}\text { Total-Ch }(\mathrm{mmol} / \mathrm{l}) \\
\text { HDL-Ch }(\mathrm{mmol} / \mathrm{l}) \\
\text { LDL-Ch }(\mathrm{mmol} / \mathrm{l}) \\
\text { TG }(\mathrm{mmol} / \mathrm{l})\end{array}$ & & \multicolumn{2}{|c|}{$\begin{array}{l}6.2 \\
0.93 \\
3.7 \\
4.65\end{array}$} & \multicolumn{3}{|c|}{$\begin{array}{l}6.1 \\
1.06 \\
4.0 \\
2.44\end{array}$} \\
\hline
\end{tabular}

Total-Ch, total cholesterol; HDL-Ch, high density lipoprotein cholesterol; LDL-Ch, low density lipoprotein cholesterol; TG, triglycerides.
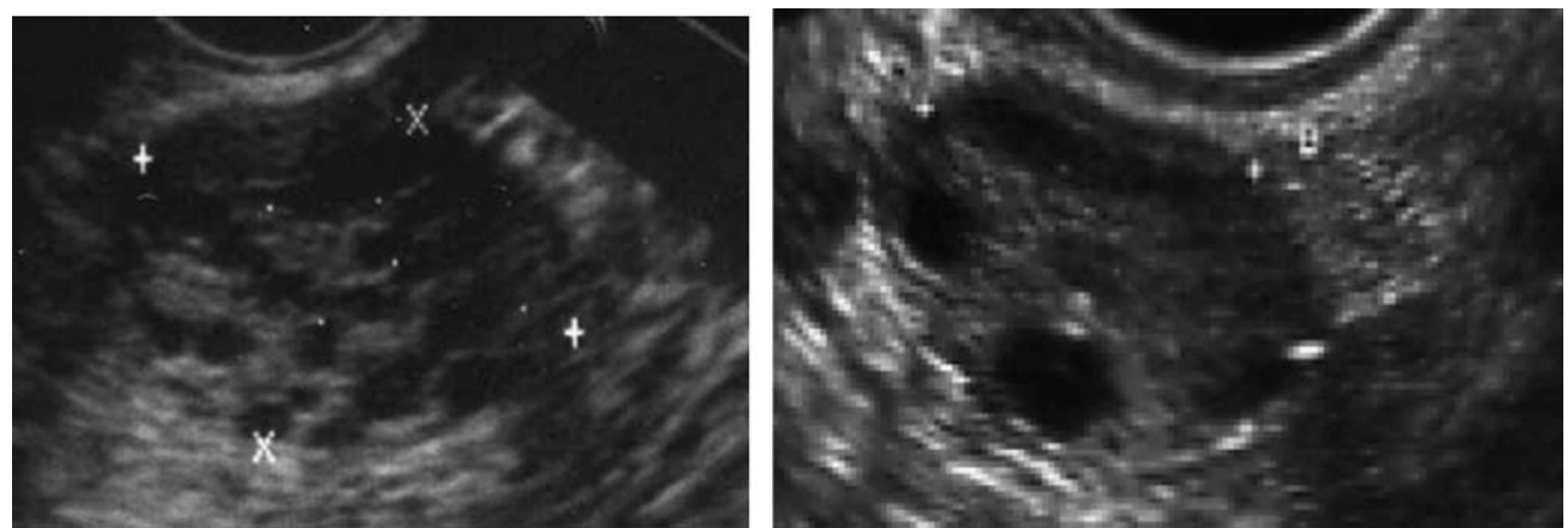

Figure 1 Polycystic appearance of the patient's right (left panel) and left (right panel) ovaries by ultrasonographic examination. Note the increased stroma in the left ovary. Ovarian volumes: right $16.0 \mathrm{ml}$, left $6.8 \mathrm{ml}$.

decreased, causing increased secretion of insulin (3). A study of obese adolescent girls with clinical features of PCOS showed increased secretion of insulin and reduced insulin sensitivity in comparison with weight-matched controls (21). Before treatment, our patient had severe hyperinsulinemia and impaired glucose tolerance, but lifestyle changes and metformin therapy ameliorated these abnormalities. A study of massively obese PCOS patients reported a high prevalence of impaired glucose tolerance and type 2 diabetes mellitus in first degree relatives, suggesting a genetic component to impaired glucose metabolism in PCOS patients (22). Interestingly, the parents of our patient had the metabolic syndrome despite being normal weight. The increased risk of metabolic impairment suggests screening in first degree relatives of patients with PCOS is warranted.

Treatment of PCOS is symptomatic. Lifestyle changes are a first-line intervention in women with PCOS, who are overweight (23). Glucose intolerance can be managed by diet and exercise, and with appropriate weight control. Metformin improves insulin sensitivity and glucose metabolism (9), and ameliorates hyperandrogenism and irregular menses in adolescents $(12,13$, $24)$. Metformin is also beneficial in normalizing the lipid profile (24). This present case clearly benefited from such treatment and no side effects were observed over a 4.5 year period. However, questions about how long treatment should be continued and long-term safety remain to be answered. Ibanez et al. have reported that the beneficial effects are lost soon after treatment is discontinued (24). It appears that PCOS is a lifelong condition. Consequently, patients should be carefully monitored during adolescence and thereafter in adulthood.

\section{Learning points}

1. PCOS is common in adolescents and should be considered in an adolescent with irregular menses and excess weight.

2. The metabolic syndrome is a common feature of PCOS. Testing for glucose intolerance and dyslipidemia is required, particularly in the presence of obesity.

3. Lifestyle changes are the first-line intervention in young women with PCOS, who are overweight

4. Management of the PCOS adolescent with metformin is beneficial and well tolerated, but the longer term effects are not yet established. 


\section{References}

1 Vural B, Caliskan E, Turkoz E, Kilic T \& Demirci A. Evaluation of metabolic syndrome frequency and premature carotid atherosclerosis in young women with polycystic ovary syndrome. Human Reproduciton 200520 2409-2413.

2 Franks S. Adult polycystic ovary syndrome begins in childhood. Best Practice and Research. Clinical Endocrinology and Metabolism $200216263-272$

3 Driscoll DA. Polycystic ovary syndrome in adolescence. Annals of the New York Academy of Sciences 2003 997 49-55.

4 Jahanfar S, Eden JA, Warren P, Seppala M \& Nguyen TV. A twin study of polycystic ovary syndrome. Fertility and Sterility 199563 478-486.

5 Legro RS, Driscoll D, Strauss JF, III, Fox J \& Dunaif A. Evidence for a genetic basis for hyperandrogenemia in polycystic ovary syndrome. Proceedings of the National Academy of Sciences of the United States of America 199895 14956-14960.

6 Battaglia C, Regnani G, Mancini F, Iughetti L, Flamigni C \& Venturoli S. Polycystic ovaries in childhood: a common finding in daughters of PCOS patients. A pilot study. Human Reproduction $200217771-776$.

7 Vink JM, Sadrzadeh S, Lambalk CB \& Boomsma DI. Heritability of polycystic ovary syndrome (PCOS) in a Dutch Twin-Family Study. Journal of Clinical Endocrinology and Metabolism, 2005.

8 Norman RJ, Wu R \& Stankiewicz MT. 4: Polycystic ovary syndrome. Medical Journal of Australia $2004180132-137$.

9 Arslanian SA, Lewy V, Danadian K \& Saad R. Metformin therapy in obese adolescents with polycystic ovary syndrome and impaired glucose tolerance: amelioration of exaggerated adrenal response to adrenocorticotropin with reduction of insulinemia/insulin resistance. Journal of Clinical Endocrinology and Metabolism 200287 1555-1559.

10 Morin-Papunen LC, Koivunen RM, Ruokonen A \& Martikainen HK. Metformin therapy improves the menstrual pattern with minimal endocrine and metabolic effects in women with polycystic ovary syndrome. Fertility and Sterility $1998 \mathbf{6 9} 691-696$.

11 Diamanti-Kandarakis E, Kouli C, Tsianateli T \& Bergiele A. Therapeutic effects of metformin on insulin resistance and hyperandrogenism in polycystic ovary syndrome. European Journal of Endocrinology $1998138269-274$.

12 Glueck CJ, Wang P, Fontaine R, Tracy T \& Sieve-Smith L. Metformin to restore normal menses in oligo-amenorrheic teenage girls with polycystic ovary syndrome (PCOS). Journal of Adolescent Health 200129 160-169.

13 Ibanez L, Valls C, Ferrer A, Marcos MV, Rodriguez-Hierro F \& de ZF. Sensitization to insulin induces ovulation in nonobese adolescents with anovulatory hyperandrogenism. Journal of Clinical Endocrinology and Metabolism 200186 3595-3598.
14 Allen HF, Mazzoni C, Heptulla RA, Murray MA, Miller N, Koenigs L \& Reiter EO. Randomized controlled trial evaluating response to metformin versus standard therapy in the treatment of adolescents with polycystic ovary syndrome. Journal of Pediatric Endocrinology and Metabolism $2005 \mathbf{1 8} 761-768$.

15 Bridger T, MacDonald S, Baltzer F \& Rodd C. Randomized placebocontrolled trial of metformin for adolescents with polycystic ovary syndrome. Archives of Pediatrics and Adolescent Medicine $2006 \mathbf{1 6 0}$ 241-246.

16 Revised 2003. Consensus on diagnostic criteria and long-term health risks related to polycystic ovary syndrome (PCOS). Human Reproduction 200419 41-47.

17 Fernandes AR, de Sa Rosa e Silva AC, Romao GS, Pata MC \& dos Reis RM. Insulin resistance in adolescents with menstrual irregularities. Journal of Pediatric and Adolescent Gynecology 2005 $18269-274$.

18 Lashansky G, Saenger P, Fishman K, Gautier T, Mayes D, Berg G, Di Martino-Nardi J \& Reiter E. Normative data for adrenal steroidogenesis in a healthy pediatric population: age- and sexrelated changes after adrenocorticotropin stimulation. Journal of Clinical Endocrinology and Metabolism 199173 674-686.

19 Pugeat M, Nicolas MH, Craves JC, varado-Dubost C, Fimbel S, Dechaud $\mathrm{H} \&$ Lejeune $\mathrm{H}$. Androgens in polycystic ovarian syndrome. Annals of the New York Academy of Sciences $1993 \mathbf{6 8 7}$ $124-135$

20 Ruutiainen K, Erkkola R, Gronroos MA \& Irjala K. Influence of body mass index and age on the grade of hair growth in hirsute women of reproductive ages. Fertility and Sterility $1988 \mathbf{5 0}$ 260-265.

21 Lewy VD, Danadian K, Witchel SF \& Arslanian S. Early metabolic abnormalities in adolescent girls with polycystic ovarian syndrome. Journal of Pediatrics 2001138 38-44.

22 Yildiz BO, Yarali H, Oguz H \& Bayraktar M. Glucose intolerance, insulin resistance, and hyperandrogenemia in first degree relatives of women with polycystic ovary syndrome. Journal of Clinica Endocrinology and Metabolism $2003 \mathbf{8 8}$ 2031-2036.

23 Norman RJ. Davies MJ, Lord J \& Moran LJ. The role of lifestyle modification in polycystic ovary syndrome. Trends in Endocrinology and Metabolism 200213 251-257.

24 Ibanez L, Valls C, Potau N, Marcos MV \& de ZF. Sensitization to insulin in adolescent girls to normalize hirsutism, hyperandrogenism, oligomenorrhea, dyslipidemia, and hyperinsulinism after precocious pubarche. Journal of Clinical Endocrinology and Metabolism 200085 3526-3530.

Received 19 June 2006

Accepted 31 July 2006 